Review

\title{
A crosstalk between p21 and UPR-induced transcription factor C/EBP homologous protein (CHOP) linked to type 2 diabetes
}

\author{
Chrysovalantou Mihailidou, Athanasios G. Papavassiliou, Hippokratis Kiaris* \\ Department of Biological Chemistry, University of Athens Medical School, 11527 Athens, Greece
}

\section{A R T I C L E I N F O}

\section{Article history:}

Received 28 August 2013

Accepted 6 November 2013

Available online 14 November 2013

\section{Keywords:}

ER-stress

UPR

Diabetes

Apoptosis

CHOP

p21

\begin{abstract}
A B S T R A C T
Type 2 diabetes (T2D) is a disease that is characterized by raised levels of glucose in the blood combined with insulin resistance and relative insulin deficiency. The pathogenesis of type 2 diabetes is associated with the induction of the unfolded protein response (UPR). While UPR aims to restore tissue homeostasis following stress of the endoplasmic reticulum (ER), prolonged ER stress triggers apoptosis at least in part through the unfolded protein response (UPR)-activated transcription factor C/EBP (CCAAT/enhancer binding protein) homologous protein (CHOP). CHOP has elevated as a critical mediator connecting accumulation and aggregation of unfolded proteins in the ER and oxidative stress and also contributes to the induction of apoptosis in $\beta$-cell (beta-cell) - cells under conditions of increased insulin demand. p21 is a cell cycle regulator that is implicated in the regulation of the UPR by various mechanisms involving inhibition of apoptosis and facilitation of the regeneration capacity of the $\beta$ cells. In this review we summarize the role of ER stress in the pathogenesis of type 2 diabetes which is associated with the induction of the unfolded protein response (UPR). We also review recent evidence associating p21 activity with $\beta$ cell health and regenerative capacity by mechanisms that may interfere with the effects of p21 in the UPR or operate independently of ER stress. Most likely understanding the molecular details of the pathogenesis of type 2 diabetes will be beneficial for the management of the disease.
\end{abstract}

(c) 2013 Elsevier Masson SAS. All rights reserved.

\section{Introduction}

Type 2 diabetes consists of an array of metabolic dysfunctions that coincide with hyperglycemia and result from the progressive

Abbreviations: T2D, type 2 diabetes; UPR, unfolded protein response; ER, endoplasmic reticulum; CHOP, C/EBP homologous protein; $\beta$-cell, beta-cell; PERK, endoplasmic reticulum kinase; eIF2, eukaryotic initiation factor-2; ATF4, activating transcription factor 4; IRE1a, inositol-requiring enzyme-1a; XBP1, X-box binding protein 1; ERAD, endoplasmic-reticulum-associated protein degradation; ATF6, activating transcription factor 6; BIP, binding immunoglobulin protein; GRP78, 78 kDa glucose-regulated protein; GRP74, 74 kDa glucose-regulated protein; PDI protein disulfide isomerase; SERCA2, sarcoplasmic ER $\mathrm{Ca}^{+2}-$ ATPase 2; NFKB, nuclear factor kappa-light-chain-enhancer of activated B cells; JNK, c-Jun, N-terminal kinase; ROS, reactive oxygen species; IR, insulin resistance; TNF-a, necrosis factor-a; IL-6, interleukin-6; CCL2, CC-chemokine ligand 2; PKC, protein kinase C; Dsba-L, disulfide-bond A oxidoreductase-like; HHcy, hyperhomocysteine; PKB, protein kinase B; SREBP-1c, sterol regulatory element-binding protein 1; VLDL, very-lowdensity lipoprotein; FFA, free fatty acids; DAG, diacylglycerol; PKB, protein kinase B; PP2A, phosphatase 2A; HFD, high-fat diet; CDK, cyclin-dependent kinases; CKIs, cyclin-dependent kinase inhibitors; HIV, human immunodeficiency virus; CMV, cytomegalovirus; Dox, doxycycline; STZ, streptozotocin; ODN, oligodeoxynucleotide; MC, mesangial cell; IGF-1, insulin-like growth factor 1.

* Corresponding author. Department of Biochemistry, University of Athens Medical School, 11527 Athens, Greece. Tel./fax: +30 2107462695

E-mail address: hkiaris@med.uoa.gr (H. Kiaris). failure of the body to secrete insulin at adequate levels or the cells ignore insulin [1]. Recent studies suggest that p21 represses $\beta$ cell duplication rate and facilitates the recovery of mice from hyperglycemia caused by streptozotocin-induced diabetes [2]. Interference with the induction of apoptosis during diabetes, such as by genetic deletion of CHOP, rescues $\beta$ cells from ER stress - related apoptosis [3]. A fundamental question regarding the decision for $\beta$ cell survival versus apoptosis is which UPR sub-pathways play a role in this transition and how the latter is being regulated. To that end it is of particular importance to understand which the key sensors of these responses are and how these responses are integrated into the cellular machinery to become protective or disadvantageous to $\beta$-cell survival upon short-term or long-term ER stress.

\section{Interplay between endoplasmic reticulum - homeostasis and protein quality control}

The endoplasmic reticulum (ER) is a highly dynamic multifold membranous structure that is responsible for post-translational folding, modification and trafficking of proteins to the cell surface or to the Golgi compartment, intracellular calcium homeostasis and lipid biosynthesis. The ER responds to genetic and environmental 
stress response and provides a catalyst proper folding environment for more or less one third of all proteins. All eukaryotic cells have evolved specific mechanisms to preserve ER functions under conditions of stress [4]. Growing evidence shows that endoplasmic reticulum (ER) stress is an important mechanism linking obesity, insulin resistance and glucose intolerance [5].

Homeostatic adaptation to endoplasmic reticulum is extremely important in insulin-secreting beta-cells because when homeostasis is disrupted, misfolded or unfolded proteins may accumulate in the ER, a condition referred to as ER stress [6]. Numerous perturbations in the normal functions of the ER such as hypoxia, alterations in calcium, nutrient availability, mutations in the ERchaperones, inhibition of protein glycosylation, reduced disulfide bond formation and viral infections, may initiate an evolutionarily conserved cellular response that is designated as the unfolded protein response (UPR). This biochemical response initially aims to restore cellular homeostasis but can eventually promote cell death if ER dysfunction is acute or prolonged [7].

The UPR is comprised of three distinct biochemical branches linked to three intracellular receptors that upon activation initiate specific biochemical events: (1) Activated PERK (endoplasmic reticulum kinase) that causes translational attenuation by phosphorylating, eIF2 (eukaryotic Initiation Factor-2). However, some specific mRNAs, including ATF4 (Activating transcription factor 4), are translated under these conditions. ATF4 can function as an activator of genes encoding adaptive functions as well as the glucose-regulated proteins [7]; (2) IRE1a (Inositol-requiring enzyme-1a) that induces the alternative splicing, and thus the activation of the transcription factor XBP1 (X-box binding protein 1) mRNA and produces XBP1-spliced (XBP1s). The activated form of XBP1 upregulates many ER chaperones and genes involved in ERAD (Endoplasmic-reticulum-associated protein degradation), various UPR 'stress genes', as well as enzymes involved in membrane biogenesis [7]; (3) ATF6 (activating transcription factor 6) that translocates to the Golgi, where it is subsequently cleaved rendering the activated form of the protein. ATF6 induces genes involved in ER homeostasis and membrane biogenesis [8].

There is an array of four types of specific cellular responses that are induced during the earlier phases of the UPR and aim to overcome stress:

1. Translational attenuation that occurs in order to overcome the load of ER and is triggered by the PERK-dependent phosphorylation of eIF2.

2. Induction of UPR-related genes, primarily chaperones such as Bip (Binding immunoglobulin protein)/GRP78 (78 kDa glucoseregulated protein) and GRP74 (74 kDa glucose-regulated protein) in order to prevent further accumulation of unfolded/ misfolded proteins.

3. Enzymes including protein disulfide isomerase (PDI) and SERCA2 (sarcoplasmic ER Ca ${ }^{+2}$-ATPase2) that increase the capacity of endoplasmic reticulum for protein folding. In addition, during UPR the transcriptional induction of genes involved in the biosynthesis of amino acids occurs, as well as the transcription of genes implicated in glutathione biosynthesis that protects against oxidative stress.

4. Stimulation of NFKB (nuclear factor kappa-light-chain-enhancer of activated $B$ cells) activity that is a transcription factor acting as a mediator of immune and anti-apoptotic response [9].

If these responses are not sufficient for the re-establishment of the cellular homeostasis, ERAD (ER-associated protein degradation) components are induced in order to eliminate the misfolded proteins [9]. Finally, if cellular damage is deemed irreversible and the pro-survival activity of the UPR is not sufficient for the retention of cellular homeostasis, apoptosis is induced by stimulation of the CCAAT/enhancer-binding homologous protein (CHOP) and activation of the JNK (c-Jun N-terminal kinase) kinase and caspase-12 [9].

\section{CHOP-dependent regulation of p21. Possible switching mechanism from the pro-survival to the pro-apoptotic response of UPR}

Obviously, there is a nonstop interplay between survival and death decisions during ER stress that determine the transition from the prosurvival towards the proapoptotic state of UPR $[10,11]$. We have provided evidence that CHOP regulates the expression of the cell cycle regulator p21 during ER stress. Pharmacological induction of ER stress by tunicamycin suppresses p21 levels, an effect that coincides with the over-expression of CHOP. CHOP suppresses $\mathrm{p} 21$ and initiates a pro-apoptotic program. Our findings indicate that CHOP relieves the anti-apoptotic activity of p21 during ER stress [12].

\section{ER stress-related mechanism of glucose-induced beta cell dysfunction}

Prolonged in vitro exposure of beta cell lines or islets to glucose increases ER stress markers in the majority of studies. This is related to the fact that increased glucose levels over-stimulate insulin production that exhausts the insulin producing and secreting activity of the $\beta$ cells leading to ER stress. The homeostatic consequences of the resulting UPR become apparent by the observation that overproduction of the ER chaperone $78 \mathrm{kDa}$ glucose-regulated protein (GRP78) partially prevents glucoseinduced beta cell dysfunction in vitro in INS-1 cells (a rat cell line that secretes insulin in response to glucose concentrations in the physiological range) [13].

\section{Evidence for a crosstalk between the generation of ROS and ER stress response}

Several experimental results suggest that the production of reactive oxygen species (ROS) results in misfolded protein-mediated cell death. Oxidative protein folding takes place in the ER implicating various oxidoreductases, including protein disulphide isomerase (PDI), PDIR, PDIp, P5 ERp57 and ERp72. In vitro formation, isomerization and reduction of disulphide bonds are catalyzed by PDI. During disulphide bond formation, cysteine residues within the PDI active site accept two electrons from the substrate resulting in the oxidation of this protein, the reduction of the PDI active site and finally in the production of hydrogen peroxide. Thus, reactive oxygen species (ROS) are produced in a cell during the synthesis of the disulphide bonds in the ER which may elicit DNA and protein damage [14]. Furthermore, glutathione is involved in the reduction of mispaired disulfide bonds that may destroy the cellular glutathione pool which is crucial for the neutralization of the reactive oxygen species (ROS) and the blockade of the oxidation of the cytosolic proteins. Considering that oxidative protein folding occurs in the ER and also that disturbances in protein folding can have damaging consequences in the cells, alterations in the redox status or the generation of ROS could directly or indirectly (or both) affect ER homeostasis and protein folding and eventually cellular function. Consequently, cells with a powerful biosynthetic load, deficient UPR, or defective ER-associated protein degradation (ERAD) are more sensitive to oxidative stress [15].

Considering that insulin biosynthesis involves disulphide bond formation oxidative stress plays a causal role in glucose-induced beta cell dysfunction both in vivo and in vitro. Analysis of clinical specimens indicates that both, ER stress and oxidative stress are 
increased in the islets of individuals with type 2 diabetes while recent studies demonstrate a close interrelationship between oxidative stress and ER-stress in beta cells [15].

\section{Contribution of ER stress in the development of metabolic actions in insulin target tissues}

A characteristic feature of T2D is the insulin resistance (IR), which occurs when cells are unable to respond to the normal behavior actions of the hormone insulin, forcing the beta cells of the pancreas to synthesize more insulin in order to address the needs for energy of the body and to maintain glucose homeostasis. This cellular stress progressively induces apoptosis of pancreatic beta cells. IR is displayed in the main target tissues of insulin, which is adipose tissue, liver and muscles. The ER stress response has strongly been implicated in the pathogenesis of diabetes [16]. ER stress affects insulin action in liver, skeletal muscles and adipose tissue as well as the function and mass of the pancreatic beta cells [17].

\subsection{Role of ER stress in adipose tissue IR}

Adipose tissue responds to metabolic and endocrine functions. Adipose tissues produce leptin and adiponectin to regulate food intake and secrete pro- and anti-inflammatory adipokines to adjust inflammatory responses and insulin resistance. Adipocytes provide fatty acids for use as an energy source in adipose tissue. Inflammatory responses in adipose tissue increase the levels of various pro-inflammatory cytokines, including the tumor necrosis factor-a (TNF-a), interleukin-6 (IL-6), IL-1b, and CC-chemokine ligand 2 (CCL2). These proinflammatory cytokines cause oxidation and endoplasmic reticulum (ER) stress, which can also provoke insulin resistance in peripheral tissues. A direct link between ER-stress and insulin signaling in adipose cells has been revealed by several studies [18].

ER stress decreases the secretion of the adipokines and consequently reduces insulin signaling and functionality in 3T3-L1 and primary mouse adipocytes without inhibiting glucose transport [19]. It has been recognized that endoplasmic reticulum dysfunction and chronic inflammation develops in adipose tissue on high fat diet mice. ER-stress is generated by free fatty acid which stimulates reactive oxygen species (ROS) and enhances proinflammatory cytokine gene expression in 3T3-L1 adipocytes. Recently it has been shown that ER stress reduces the expression of the lipid protein, perilipin $A$ and enhances lipolysis in adipose cells. Inducers of ER-stress activate a lipolytic reaction. The consequences of ER stress on adipose lipolysis were not reversed by inhibitors of several stress-activated kinases such as JNK, p38MAPK and PKC (Protein kinase $\mathrm{C}$ ), kinases that are usually involved in the negative regulation of insulin signaling [20]. These data support a role of ERstress and modulation of inflammatory responses and in lipid homeostasis.

ER stress inducers enhance a decrease of both leptin and adiponectin secretion and an increase of IL-6 secretion [18]. The decrease of the expression of ER disulfide-bond A oxidoreductaselike (Dsba-L) weakens adiponectin folding and multimerization, leading to ER stress [21,22]. In addition, ER stress, through upregulation of CHOP, reduces resistin (adipose tissue-specific secretory factor transcription in adipocytes) [23].

Recent data showed that hyperhomocysteine (HHcy) induces insulin resistance in adipose tissue which in turn promotes insulin resistance. Variant ER stress inducers were increased in adipose tissue of HHcy mice. HHcy activates c-Jun N-terminal kinase (JNK), contributed in insulin resistance by inhibiting protein kinase $\mathrm{B}$ (PKB) activation. Furthermore, JNK activated c-Jun and p65, which stimulated the transcription of pro-inflammatory cytokines [24]. Collectively these results indicate that ER stress is an important player in adipose tissue dysfunction and promotes IR.

\subsection{Role of ER stress in liver IR}

The liver is the main site of the Novo lipogenesis, which allows the synthesis of fatty acids from excess carbohydrates in the diet. Liver cells are the main key players of lipogenesis and glucose metabolism. Various data have suggested that endoplasmic reticulum (ER) stress could involve in fatty acid synthesis and cholesterol metabolism [17]. SREBP-1c (sterol regulatory element-binding protein 1) which is an activator of lipogenic gene transcription is activated by ER-stress. During prolonged ER stress GRP78 is released from SREBP-1c by unmasking the Golgi localization signal on SREBP-1c, which then migrates to the Golgi apparatus where SREBP-1c is proteolytically processed to yield an active fragment that enters to the nucleus and stimulates lipogenic genes. This processing is activated by site 1 and site 2 proteases in the Golgi [25]. Reduced VLDL (Very-low-density lipoprotein) secretion and increased SREBP-1c activation in combination with reduced lipid oxidation aggravate lipogenesis and initiate to a vicious circle leading to steatosis [26]. In hepatocytes XBP1s targets mRNAs implicated in fatty acid synthesis. Indeed, a recent study showed that liver-specific XBP1-null mice failed to develop steatosis when placed to a high carbohydrate diet [27]. Furthermore, induction of ER stress/UPR activation tunicamycin showed a remarkable downregulation of key lipid metabolic enzymes including CEBP $\alpha$. Interestingly tunicamycin treatment in livers of mice deleted ATF6, IRE1, p58IK showed lack of expression of genes involved in metabolism correlated to livers from wild-type mice. However over-expression of $\mathrm{CHOP}$ under tunicamycin treatment preserves the metabolic gene expression and decreased hepatic steatosis in mice on a high fat diet. So CHOP plays a key role in sustaining metabolism gene under prolonged ER-stress and acts as a possible mediator of proapoptotic and pro-inflammatory properties [28].

\subsection{Role of ER stress in muscle IR}

High levels of circulating fatty acids in muscle cause insulin resistance in type 2 diabetes. Several studies have reported an involvement with increased plasma FFA (free fatty acids) levels by lipid infusion leads to insulin resistance whereas lowering chronically elevated plasma FFA levels improves insulin sensitivity in rodents and humans [29]. Generation of lipids, such as diacylglycerol (DAG), fatty acyl-CoA and ceramides modify insulin response and consequently lead to defects in glucose metabolism and insulin resistance [30]. Recent studies have suggested that endoplasmic reticulum (ER) stress seems to be one of the major mechanisms involved in free fatty acid-induced insulin resistance [31]. Increased ceramide accumulation was reported in skeletal muscle of insulin resistance rodents $[32,33]$ and in muscles of obese insulin-resistant patients [34]. They seem to be the most potent inhibitors of insulin signaling in muscle cells [35]. Saturated fatty acids, such as the accumulation of palmitate in muscle cells, induce ceramide biosynthesis at the level of the endoplasmic reticulum (ER) [36]. Increased levels of ceramides inhibit the insulin signaling pathway through the inactivation of protein kinase $B$ (PKB) via mechanisms involving both protein kinase $C$ (PKC) and protein phosphatase 2A (PP2A) [37]. Studies have reported the role of ER stress to mediate palmitate-induced IR in muscle cells. These have shown that direct exposure of human primary myotubes, $\mathrm{C} 2 \mathrm{C} 12$ myotubes [36], or L6 myotubes [38] to palmitate is capable of stimulate ER stress. Similar results were studied in vivo from rats 
fed a high-fat diet (HFD) [36]. Together, these data suggest that muscle IR may be linked to ER stress activation.

\section{Pancreatic beta cells: relationship between survival signaling and apoptosis signaling}

Many genetic and environmental variations trigger apoptotic pathways that eventually eliminate damaged cells. The decision for a protective or destructive stress response to cells depends on the nature and the extent of the stress as well as the subjected specific cell type [39].

Beta-cells represent a specialized type of cells in the pancreas that are localized in the islets of Langerhans constituting $65-80 \%$ of the cells in the islets [40]. Pancreatic beta cells, are the only source of insulin in the body and are either lost or become dysfunctional during the progression of diabetes that leads to the increase in the glucose levels in the blood. Targeting, and restoring the function of beta cells constitutes a major therapeutic strategy for the treatment of diabetes [40]. Another important type of endocrine cells in the pancreas is the alpha cells (15-20\%) which produce glucagon and elevates the glucose levels in the blood [41]. Beta cell mass is dynamically regulated and is maintained through a delicate balance of regeneration and apoptosis [42].

A significant increase in beta cell number develops under conditions that include obesity and insulin resistance in order to cope with the elevated demands for insulin production. The increase in beta cell mass can occur through an increase in the cell number by neogenesis and proliferation (hyperplasia), or through an increase in the cell size (hypertrophy). Pancreatic $\beta$-cell mass is regulated by interference with at least four independent mechanisms that contribute to the emergence and function of $\beta$ cells that are responsible to cope with conditions related to the increased demand for insulin production. Those include the induction of $\beta$ cell replication, the increase in $\beta$ cell size, the de novo production of $\beta$ cells (neogenesis) and finally the inhibition of $\beta$ cell apoptosis [43].

\section{Wolfram syndrome and $\beta$ cell dysfunction}

Hereditary syndromes such as the Wolfram syndrome illustrate the intrinsic link between ER stress and diabetes. Wolfram syndrome is a neurodegenerative disorder that is characterized by diabetes mellitus caused by non-autoimmune loss of beta cells, deafness and optic atrophy [44]. Wolfram syndrome is activated by mutations in the $W f s 1$ gene $[45,46]$. Despite that WFS1 is not a direct marker of the UPR, analysis of Wfs1-deficient mice demonstrates that WFS1 function is linked with the preservation of ER homeostasis. WFS1 is induced by XBP1 through a response element-like motif in SH-SY5Y cells. In mice the pancreatic $\beta$ cells are subjected to ER-stress as shown by the phosphorylation of eIF2 and the production of the spliced form of XBP1. Mutations in Wfs1 repress intracellular calcium signaling, beyond glucose stimulation associated with insufficient insulin secretion and activate UPRregulated genes, while ultimately inhibits cell cycle and stimulates apoptosis [44-47]. WFS1 interferes with the maturation of plasma membrane proteins or the stability of ER membrane proteins. The finding that WFS1 and $\mathrm{Na}(+) / \mathrm{K}(+)$ ATPase $\beta 1$ proteins interact physically implies an important role for WFS1 in this ATPase function. Decreased levels of this ATPase subunit were identified in the fractions of plasma membrane of Wfs 1 mutant cells and of Wfs1 knockdown cells [48]. Accordingly loss of function in the WFS1 induces ER stress-cell failure associated with diabetes. Thus, WFS1 is involved in membrane trafficking, protein processing and/or regulation of ER calcium homeostasis [48]. This discovery suggested that WFS1 has a protective role against ER stress and reveals the consequences of the negative regulation of pancreatic beta cell death in the pathogenesis of diabetes.

\section{CDKs, regulators of the cell cycle}

Cell-cycle progression is orchestrated by a series of events at which cyclins and their partners, the cyclin-dependent kinases (CDK) play a fundamental role. The activation of the kinase activity of the CDKs is responsible for the sequential progression towards the phases of the cell cycle. p21 is a member of the CIP/Kip family of cyclin-dependent kinase inhibitors (CKIs). This family includes p27 and p57 [49]. These CKIs contain a region at the $\mathrm{N}$ terminal transcriptional activation domain ( $\mathrm{NH} 2$ terminus) that is crucial for the inhibition of cyclin/Cdk complexes, while the $\mathrm{COOH}$ terminal regions are changeable in length and function [50]. CKIs bind and inhibit a wide range of cyclin/Cdk complexes, especially those containing Cdk2 [51]. p21 expression up-regulates tumor-associated genes and proteins implicated in age-related diseases [52] and stimulates viral promoters, including those of HIV (Human immunodeficiency virus) and CMV (Cytomegalovirus) [53,54]. The expression of $\mathrm{p} 21$ is regulated by $\mathrm{p} 53$ (tumor suppressor protein 53), through which p21 mediates the p53-dependent cell cycle arrest in response to a variety of stress-inducing conditions. In parallel with its cytostatic function as an inhibitor of cell cycle progression p21 also operates as an inhibitor of apoptosis exhibiting a pro-survival activity as it protects cells from p53-dependent and p53-independent apoptosis [55]. This dual activity of p21 to regulate both cell cycle and susceptibility to apoptosis appears to play a regulatory role in the transition of UPR from its prosurvival towards its proapoptotic mode of action [12].

The role of p21 in the regulation of other cellular activities beyond the direct regulation of the cell cycle has recently attracted increasing attention. Recent findings indicate that p21 is essential for damage-induced paracrine antiapoptotic activity because of the ability of p21 to imitate a transcriptional damage response without DNA damage. p21 interacts with CDK8 that is a CDK family member regulating several transcriptional programs involved in carcinogenesis but has no role in cell cycle progression. Recent evidence showed that pharmacological inhibition of CDK8 impeded different chemotherapy-induced tumor-promoting paracrine functions of normal and tumor cells, in vitro and in vivo [56].

\section{Maintenance of glucose homeostasis in the p21-null mouse}

The ability of p21 to regulate both cell cycle and sensitivity against apoptosis-inducing signals [57], in combination of various lines of evidence pointing to a role of p21 in maintaining stem cell function $[58,59]$ prompted several investigators to explore a potential association between $\mathrm{p} 21$ and diabetes.

The onset of oxidative stress during diabetes stimulated the performance of studies assessing the role of p21 in the glucoserelated toxicity. Isolated rat pancreatic islet cells were treated with $\mathrm{H}_{2} \mathrm{O}_{2}$ to provoke oxidative stress. Also in Zucker diabetic fatty rats p21 was overexpressed in islet cells using an adenovirus. Analysis of gene expression in both experiments suggested that p21 mRNA expression was elevated whereas insulin mRNA was diminished. These findings support the notion that the expression of $\mathrm{p} 21$, which can be induced by oxidative stress, aggravates in pancreatic islet cells upon development of diabetes and suggest that by inhibiting cell proliferation and insulin biosynthesis, the induction of p21 is likely possible to be concerned in the beta cell glucose toxicity [60].

In vitro experiments showed that p21-deficient mouse islets treated with beta cell mitogens exhibited higher rates of DNA synthesis as compared to p21-expressing islets which is in line with 
the role of p21 in inhibiting cell cycle progression [60]. However, in vivo studies conducted by the same research group in $\mathrm{p} 21$ deficient mice demonstrated that beta cell function and replication rate, as well as glucose homeostasis are not altered as compared to the controls. This result suggests that either p21 does not act alone to arrest the beta cell cycle or that its function is complemented by another protein that can substitute for the loss of p21 in b cells in vivo [61]. Interestingly though, in this study, p21 deficient mice displayed lower insulin levels than controls implying some dysfunction of the $b$ cells which however did not become clinically relevant, at least under the conditions used by the investigators in this study. It is conceivable though that p21 deficiency might have sensitized mice to the harmful consequences of ER stress at levels below those that could interfere with glucose homeostasis. This is in line with recent findings showing that p53deficient cells and animals, that have reduced p21 levels, are more sensitive to chronic and acute ER stress [62].

\section{Over-expression of p21 in $\beta$-cells inhibits cell proliferation}

Beta cell proliferation is a very important contributor to the dynamic nature of adult beta cell mass [63]. p21 functions as an inhibitor of the cell cycle progression, however, p21 ablation in islets has no response on islet mass and glucose metabolism [64]. To determine whether $\mathrm{p} 21$ contributes to the $\beta$-cell failure associated with type 2 diabetes, the effects of $\mathrm{p} 21$ in the pancreatic $\beta$-cell regeneration were examined by an alternative approach. Specifically, the consequences of conditional p21 over-expression in $\beta$ cell proliferation were investigated by using a tetracycline-based inducible system (Insulin-rtTA/TET-p21). In this system p21 is controlled by the RIPII-rtTA promoter and therefore it was targeted in the pancreatic $\beta$-cells. Therefore, following doxycycline (Dox) administration the specific over-expression of p21 in islet $\beta$ cells was induced, which can inhibit the proliferation of $\beta$ cells. Chronic administration of doxycycline in these mice slowly elevated serum glucose levels and eventually caused diabetes that apparently was associated with the reduction in b-cell content and insulin production [2].

11.1. Over-expression of p21 in mouse islets improves the recovery from streptozotocin-induced diabetes

In the same study the recovery of the b-cells from streptozotocin (STZ)-induced toxicity was also evaluated. STZ has the preferential toxicity to pancreatic $\beta$ cells and is known to induce insulindependent diabetes, by eliciting damage in the beta cells of the experimental animals [65]. Diabetes induction was accomplished by utilizing a single high STZ dose, to reach toxicity to pancreatic $\beta$ cells. A single injection of a high dose of STZ, at $200 \mathrm{mg} / \mathrm{kg}$, was administered intra-peritoneal in mice on the eighth day of Dox treatment and blood glucose levels were measured. Surprisingly, the p21-overexpressing transgenic mice promoted the recovery from STZ injury. Also p21-overexpressing mice were far more capable to increase weight and attain normal glucose homeostasis in the blood than control littermates. This demonstrates that when b-cell self-duplication is repressed, such as when p21 is overexpressed, islet damage from STZ treatment may re-activate alternative mechanisms to induce efficiently $\beta$-cell regeneration for diabetic recovery [2].

\subsection{Structural changes in islets in p21-overexpressing transgenic mice}

In order to determine which cells in the islets are responsible for $\beta$ cell regeneration morphometric analysis identified a network operated by the transcription factors Ngn3 (Pax4, Arx, Nkx2.2, NeuroD1 and Pax6) and putative progenitor markers CD133 and cMet, to be expressed in the islets of transgenic mice that overexpress p21 following STZ treatment and stimulated islet injury. Nonetheless, the expression of these genes was not detectable in the islets of control mice directly after STZ injury. These results highlight that the progenitor cell stimulation could be promoted under conditions where b-cell self-renewal conditions are inhibited [2], illustrating the role of $\mathrm{p} 21$ in $\beta$ cell function.

\section{3. p21-Overexpressing transgenic mice co-expressing endocrine hormones}

After STZ treatment, the levels of endocrine markers such as glucagon, insulin and somatostatin were increased [2] while newly differentiated endocrine cells express insulin, glucagon, and somatostatin spontaneously during early pancreas development $[66,67]$. The progenitor islet cells of STZ-double transgenic mice coexpress insulin, glucagon and somatostatin in much higher levels than the untreated double transgenic mice. Therefore diabetic recovery in p21 transgenic mice treated with streptozotocin, are characterized by regeneration in the adult beta islets and this might be the answer of the possibly activation of early pancreatic developmental pathways [2].

\section{The biological significance of p21 associated with diabetic nephropathy}

Renal mesangial cell hypertrophy is a characteristic feature of diabetic nephropathy which acts as a reaction to renal stress or injury and is defined by increased protein synthesis. Considering that p21 controls cell cycle progression and affects DNA replication while allowing protein synthesis to continue, the implication of p21 in renal mesangial hypertrophy was considered. p21 inhibition by specific phosphorothioate antisense oligodeoxynucleotide (ODN) decreases p21 protein levels in human mesangial cell (MC). It is known that IGF-1 plays important roles in cellular functions including cell proliferation related to hypertrophy. In addition, antisense p21 ODN caused attenuation of IGF-1 (Insulin-like growth factor 1)-induced p21 levels of MC cells and resulted in attenuating the hypertrophic effect. These data suggest that attenuation of p21 may ultimately prove useful in the therapy of glomerular hypertrophic diseases [68].

\section{ER stress-associated apoptosis factor CHOP associated with type 2 diabetes}

CCAAT/enhancer binding protein (C/EBP) homologous protein (CHOP)/growth arrest and DNA damage 153 is a dominant-negative member of the C/EBP transcription family. CHOP is a leucine zipper transcription factor that is present mostly in the cytosol, at low levels under normal conditions. Induction of CHOP after stress conditions accumulates in the nucleus [69]. CHOP was identified as an ER stress transcription factor that induces apoptosis in response to ER stress. CHOP expression is strongly induced via IRE1 and PERK signaling $[12,69]$. The proapoptotic effect of CHOP that is transduced by Bim $[70,71]$ enhances when ER stress cannot be restrained by the prosurvival action of the response system, and the levels of misfolded proteins remain elevated. In this response, CHOP enhances a transcription program that promotes the induction of apoptosis. This includes expression of the proapoptotic Bim and repression of the anti-apoptotic Bcl-2 (B-cell lymphoma 2). CHOP also induces death receptor 5 (DR5), which further leads cells to undergo apoptosis by multiple conditions that cause ER stress [72]. 


\section{Importance of chronic ER stress - mediated $\beta$ cell apoptosis in AKITA mouse}

The Akita mouse have a point mutation in the insulin 2 gene (Ins2) which carry the Cys96Tyr which results in disrupting a disulfide bond between A and B chains and causes a drastic conformation change of this molecule. Therefore, mutant insulin is not secreted and is degraded by the ERAD pathway that is associated with the induction of ER-stress, which in turn causes $\beta$ cell dysfunction and finally apoptosis [71,73].

\section{Role of CHOP deletion to the homozygous Ser51Ala mutant eIF2a}

Accumulation of unfolded proteins within the endoplasmic reticulum (ER) decreases mRNA translation via PERK-mediated phosphorylation of eukaryotic initiation factor 2 on the Ser 51 residue of the a subunit (eIF2a). Diabetes associated with the loss of PERK activity is speculated to be due to a postnatal loss of $\beta$ cells through apoptotic cell death arising from a failure to properly regulate the endoplasmic reticulum unfolded protein response (UPR) [74]. PERK deficiency may have a crucial role impact on physiological conditions associated with ER stress [75].

To elucidate the role of eIF2a phosphorylation, homozygous Ser51Ala mutant eIF2a mice were generated. Mice homozygous for the mutation at the eIF2 $\alpha$ phosphorylation site (Ser51Ala) exhibited a neonatal lethal phenotype associated with accumulation of misfolded proteins and defective transport, oxidative stress related to mitochondrial damage, decreased expression of UPR, anti-oxidant and beta cell-associated genes, and finally with defective gluconeogenesis related to severe $\beta$-cell deficiency. These results indicate that the UPR has an extensive role than preserving functional ER protein than originally anticipated and is essential for the in vivo glucose homeostasis by various mechanisms [76].

\subsection{Role of CHOP deletion to the heterozygous Ser51Ala mutant eIF2a}

While heterozygous $e I F 2 \alpha^{s / A}$ animals did not spontaneously manifest $\beta$-cell failure, upon feeding them with a $45 \%$ high-fat diet, these mice developed diabetes characterized by increased fasting blood glucose, glucose intolerance and a $\beta$-cell secretion deficiency. Defective insulin secretion was due to an elevated rate of glucosestimulated translation that compromised the activity of the unfolded protein folding response and led to the distention of the ER compartment, the prolonged association of misfolded proinsulin with the ER chaperone BiP and finally with the reduced secretory granule content [3].

These findings demonstrated that regulation of mRNA translation phosphorylation of eIF $2 \alpha$ is crucial for the maintenance of functional integrity of endoplasmic reticulum [3]. While eIF2 phosphorylation inhibits protein translation, it is required for the transcriptional induction of the majority of the ER stress-inducible genes associated with the UPR, including those encoding for the glucose-regulated proteins, such as GRP78/BiP, ERp72, GRP94, and CHOP. CHOP expression is increased in $\beta$ cells from diabetic mice and humans. As ER distention and $\beta$-cell death in homozygous Ser51Ala eIF2 $\alpha$ islets were apparent, it is evident that there are

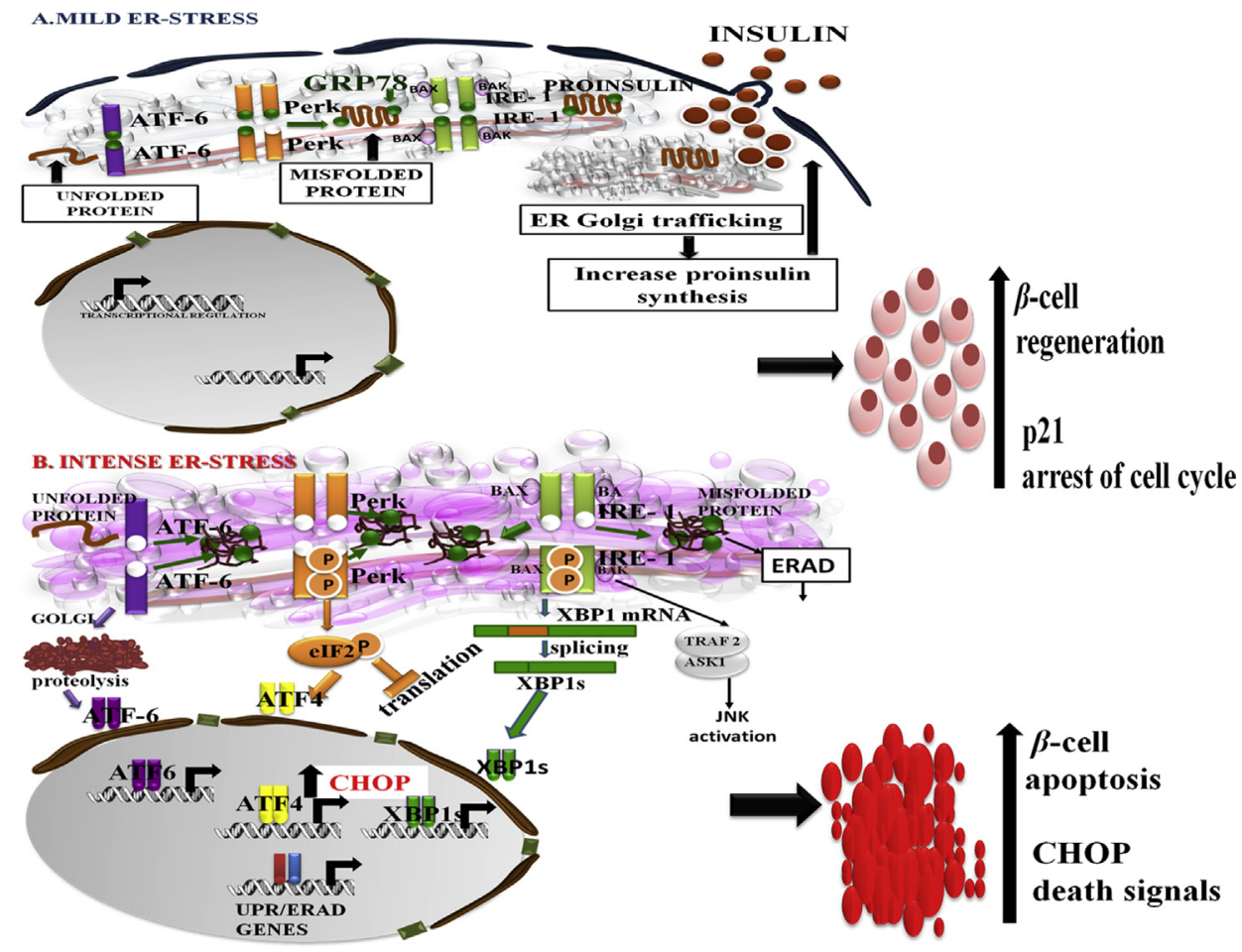

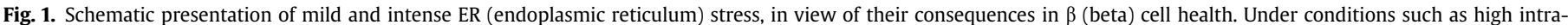

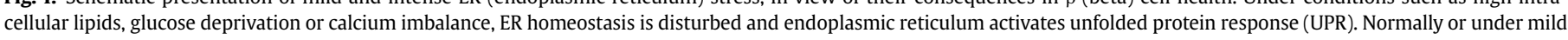

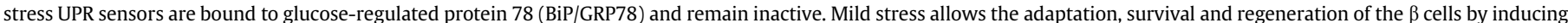

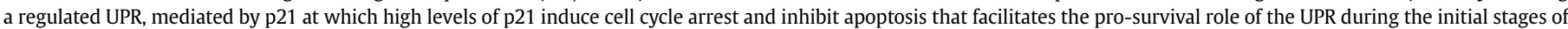

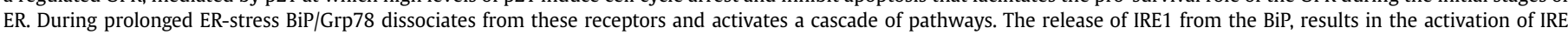

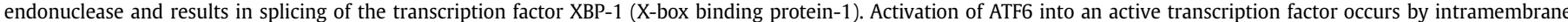

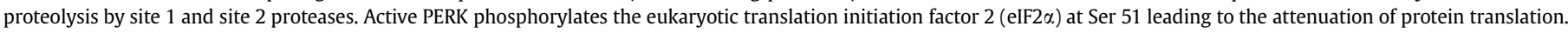
Moreover, phosphorylation of eIF2 $\alpha$ induces translation of ATF4 and its target C/EBP-homologous protein (CHOP) which inhibits p21 transcription and stimulates apoptosis. 
environmental stimuli that induce eIF $2 \alpha$ phosphorylation early in development that is fundamental for $\beta$-cell survival [77]. The requirement of beta cell for eIF $2 \alpha$ phosphorylation is not mediated through Atf4 mRNA because mice lacking ATF4 have no reported defects in glycemic control [78]. $\beta$ cells from mice with homozygous Ser51Ala eIF2 $\alpha$ underwent apoptosis in part signaled through CHOP. However in CHOP-null mice with heterozygous Ser51Ala eIF2 $\alpha$ mutations $\beta$ cell mass was increased, $\beta$ cell function was improved, and glucose intolerance was prevented compared with islets from Ser51Ala eIF2 $\alpha$ mice with wild type CHOP alleles [3]. These data support the notion that CHOP is involved in apoptosis of $\beta$ cells. Nevertheless, CHOP is not the exclusively death signal elicited by ER stress, in the absence of $\mathrm{CHOP}$ other apoptotic pathways are evoked. CHOP deletion prevents $\beta$ cell apoptosis and improves $\beta$ cell function by preventing oxidative damage and preserves homeostasis in ER [3].

In another experiment, eIF $2 \alpha^{\mathrm{s} / \mathrm{s}}$ and eIF $2 \alpha^{\mathrm{s} / \mathrm{A}}$ mice were fed with $45 \%$ HF (high fat) diet for 35-41 weeks. Body mass, glucose tolerance and levels of serum insulin were examined. In this model CHOP deletion increased obesity, and the number of functional $\beta$ cells as a consequence of preventing glucose intolerance [3] also implying the role of $\mathrm{CHOP}$-dependent apoptosis in $\mathrm{b}$ cells diabetes.

\subsection{Effect of CHOP-null mutation in a HF diet streptozotocin treated model of T2D}

Wild-type and CHOP-null mice were treated with STZ and were fed with a high cholesterol $60 \%$ HF diet for 5-6 weeks. The results indicated that in wild type mice $\beta$ cell mass was reduced and animals developed hyperglycemia. However in CHOP-null mice improvement of $\beta$ cell function was observed and glucose homeostasis was maintained. The findings also indicate that the CHOPnull mutation in a HF diet-fed, STZ-treated non-genetic model of T2D has an impact to $\beta$ cell function and recovery [3].

\subsection{Effect of CHOP-null mutation in Lepr ${ }^{d b / d b}$ mice}

Leptin deficient mice $(\mathrm{db} / \mathrm{db})$ mice are characterized by insulin resistance, are hypertriglyceridemic, and exhibit impaired glucose tolerance. Accumulated levels of UPR markers were identified in the islets of $d b / d b$ mice [77,79].

Furthermore, in order to investigate whether $\mathrm{CHOP}$ is involved in the maintenance of functional $\beta$ cells, heterozygous for leptin mice and leptin-deficient mice were studied. CHOP deletion in the leptin-null mice upregulates expression of UPR and anti-oxidative response markers and decreases expression of proapoptotic genes. This in turn results in delayed glucose tolerance, elevated $\beta$ cell proliferation and prevention of $\beta$ cell apoptosis [3].

\subsection{CHOP deficiency protects $\beta$ cells from oxidative stress related to ER-stress}

Islets from CHOP-deficient or wild type mice were treated with tunicamycin to block $\mathrm{N}$-linked glycosylation of proteins and to promote ER-stress. These experiments showed that there was an increase in the oxidative degradation of proteins and lipids in wildtype islets [3]. Alternatively, treatment of islets from wild type and $\mathrm{CHOP}^{-1-}$ mice with $\mathrm{H} 2 \mathrm{O} 2$, showed the presence of comparable amounts of oxidative damage in the islets from $\beta$-cells from wild type and CHOP null mice [3]. Consequently, CHOP deletion decreased the oxidative damage that results from the unfolded protein response in the ER, but not from general oxidative stress. These findings demonstrated that CHOP deletion improves $\beta$ cell function and prevents apoptosis by improving the capacity of ER to produce folded proteins [3].

\section{Conclusions}

The cellular and molecular mechanisms governing ER-stress is fundamental for understanding the consequences caused by type 2 diabetes. Growing evidence indicates that hyper-activation of the UPR is indispensable for ER homeostasis and has a role in $\beta$ cell failure and dysfunction during diabetes and place the unfolded protein response as an important mediator in the development of the disease and the manifestation of its complications. Paradoxically there are interrelationships between type 2 diabetes and the balance between survival pathway and apoptosis pathway related to ER-stress. It is currently believed that over-expression of p21 is responsible for the recovering of STZ-induced diabetes so it is implicated in the prosurvival effects of diabetes-related UPR. Alternatively CHOP deletion determines the transcriptional profile of the cell to conserve the functional capacity of the ER and downsize accumulation of ROS, so it engages the apoptotic pathway (Fig. 1). The potential link between $\mathrm{p} 21$ and CHOP regulation imply novel therapeutic interventions as well as better understanding of basic aspects of $\beta$ cell biology.

\section{Acknowledgments}

Ch.M. has been supported by a Fellowship that is co-financed by the European Union (European Social Fund - ESF) and Greek national funds through the Operational Program "Education and Lifelong Learning" of the National Strategic Reference Framework (NSRF) - Research Funding Program: Heracleitus II. Investing in knowledge society through the European Social Fund.

\section{References}

[1] S. Kataoka, J. Satoh, H. Fujiya, T. Toyota, R. Suzuki, K. Itoh, K. Kumagai, Immunologic aspects of the nonobese diabetic (NOD) mouse. Abnormalities of cellular immunity, Diabetes 32 (1983) 247-253.

[2] J. Yang, W. Zhang, W. Jiang, X. Sun, Y. Han, M. Ding, Y. Shi, H. Deng, p21cipoverexpression in the mouse beta cells leads to the improved recovery from streptozotocin-induced diabetes, PLoS One 4 (2009).

[3] B. Song, D. Scheuner, D. Ron, S. Pennathur, R.J. Kaufman, Chop deletion reduces oxidative stress, improves $\beta$ cell function, and promotes cell survival in multiple mouse models of diabetes, J. Clin. Invest. 118 (2008) 3378-3389.

[4] C. Sidrauski, R. Chapman, P. Walter, The unfolded protein response: an intracellular signalling pathway with many surprising features, Trends Cell Biol. 8 (1998) 245-249.

[5] D. Scheuner, R.J. Kaufman, The unfolded protein response: a pathway that links insulin demand with beta-cell failure and diabetes, Endocr. Rev. 29 (2008) 317-333.

[6] C.J.H. Van Der Kallen, M.M.J. Van Greevenbroek, C.D.A. Stehouwer, C.G. Schalkwijk, Endoplasmic reticulum stress-induced apoptosis in the development of diabetes: is there a role for adipose tissue and liver? Apoptosis Int. J. Program. Cell Death 14 (2009) 1424-1434.

[7] D. Ron, P. Walter, Signal integration in the endoplasmic reticulum unfolded protein response, Nat. Rev. Mol. Cell Biol. 8 (2007) 519-529.

[8] H. Yoshida, T. Matsui, A. Yamamoto, T. Okada, K. Mori, XBP1 mRNA is induced by ATF6 and spliced by IRE1 in response to $\mathrm{ER}^{3}$ stress to produce a highly active transcription factor, Cell 107 (2001) 881-891.

[9] S. Oyadomari, M. Mori, Roles of CHOP/GADD153 in endoplasmic reticulum stress, Cell Death Differ. 11 (2004) 381-389.

[10] E. Szegezdi, S.E. Logue, A.M. Gorman, A. Samali, Mediators of endoplasmic reticulum stress-induced apoptosis, EMBO Rep. 7 (2006) 880-885.

[11] A.H. Schönthal, T.C. Chen, F.M. Hofman, S.G. Louie, N.A. Petasis, Preclinical development of novel anti-glioma drugs targeting the endoplasmic reticulum stress response, Curr. Pharm. Des. 17 (2011) 2428-2438.

[12] C. Mihailidou, I. Papazian, A. Papavassiliou, H. Kiaris, CHOP-dependent regulation of p21/waf1 during ER stress, Cell. Physiol. Biochem. (2010) 761-766.

[13] T. Hartley, M. Siva, E. Lai, T. Teodoro, L. Zhang, A. Volchuk, Endoplasmic reticulum stress response in an INS-1 pancreatic $\beta$-cell line with inducible expression of a folding-deficient proinsulin, BMC Cell Biol. 11 (2010) 59.

[14] C.X. Santos, L.Y.Tanaka, J. Wosniak, F.R. Laurindo, Mechanisms and implications of reactive oxygen species generation during the unfolded protein response: roles of endoplasmic reticulum oxidoreductases, mitochondrial electron transport, and NADPH oxidase, Antioxid. Redox Signal. 11 (2009) 2409-2427.

[15] J.D. Malhotra, R.J. Kaufman, Endoplasmic reticulum stress and oxidative stress: a vicious cycle or a double-edged sword? Antioxid. Redox Signal. 9 (2007) 2277-2293. 
[16] M. Flamment, E. Hajduch, P. Ferré, F. Foufelle, New insights into ER stressinduced insulin resistance, Trends Endocrinol. Metab. TEM 23 (2012) 1-10.

[17] G.S. Hotamisligil, Endoplasmic reticulum stress and the inflammatory basis of metabolic disease, Cell 140 (2010) 900-917.

[18] L. Xu, G.A. Spinas, M. Niessen, ER stress in adipocytes inhibits insulin signaling, represses lipolysis, and alters the secretion of adipokines without inhibiting glucose transport, Horm. Metab. Res. Horm. Und Stoffwechselforsch Horm. Metab. 42 (2010) 643-651.

[19] K.T. Pfaffenbach, C.L. Gentile, A.M. Nivala, D. Wang, Y. Wei, M.J. Pagliassotti, Linking endoplasmic reticulum stress to cell death in hepatocytes: roles of C/ EBP homologous protein and chemical chaperones in palmitate-mediated cell death, Am. J. Physiol. - Endocrinol. Metab. 298 (2010) 1027-1035.

[20] J. Deng, S. Liu, L. Zou, C. Xu, B. Geng, G. Xu, Lipolysis response to endoplasmic reticulum stress in adipose cells, J. Biol. Chem. 287 (2012) 6240-6249.

[21] M. Liu, L. Zhou, A. Xu, K.S.L. Lam, M.D. Wetzel, R. Xiang, et al., A disulfide-bond A oxidoreductase-like protein (DsbA-L) regulates adiponectin multimerization, Proc. Natl. Acad. Sci. U. S. A. 105 (2008) 18302-18307.

[22] L. Zhou, M. Liu, J. Zhang, H. Chen, L.Q. Dong, F. Liu, DsbA-L alleviates endoplasmic reticulum stress-induced adiponectin downregulation, Diabetes 59 (2010) 2809-2816.

[23] M.I. Lefterova, S.E. Mullican, T. Tomaru, M. Qatanani, M. Schupp, M.A. Lazar, Endoplasmic reticulum stress regulates adipocyte resistin expression, Diabetes 58 (2009) 1879-1886.

[24] Y. Li, H. Zhang, C. Jiang, M. Xu, Y. Pang, J. Feng, et al., Hyperhomocysteinemia promotes insulin resistance by inducing endoplasmic reticulum stress in adipose tissue, J. Biol. Chem. (2013) 1-22.

[25] N.E. Wolins, D.L. Brasaemle, P.E. Bickel, A proposed model of fat packaging by exchangeable lipid droplet proteins, FEBS Lett. 580 (2006) 5484-5491.

[26] S. Basseri, R.C. Austin, Endoplasmic reticulum stress and lipid metabolism: mechanisms and therapeutic potential, Biochem. Res. Int. 2012 (2012) 841362.

[27] H.K. Lee, J.M. Lund, B. Ramanathan, N. Mizushima, A. Iwasaki, Autophagydependent viral recognition by plasmacytoid dendritic cells, Science 315 (2007) 1398-1401.

[28] S. Oyadomari, H.P. Harding, Y. Zhang, M. Oyadomari, D. Ron, Dephosphorylation of translation initiation factor 2alpha enhances glucose tolerance and attenuates hepatosteatosis in mice, Cell Metab. 7 (2008) 520-532.

[29] E. Qvigstad, I.L. Mostad, K.S. Bjerve, V.E. Grill, Acute lowering of circulating fatty acids improves insulin secretion in a subset of type 2 diabetes subjects, Am. J. Physiol. - Endocrinol. Metab. 284 (2003) 129-137.

[30] E.W. Kraegen, G.J. Cooney, Free fatty acids and skeletal muscle insulin resistance, Curr. Opin. Lipidol. 19 (2008) 235-241.

[31] U. Ozcan, Q. Cao, E. Yilmaz, A.H. Lee, N.N. Iwakoshi, E. Ozdelen, et al., Endoplasmic reticulum stress links obesity, insulin action, and type 2 diabetes, Science 306 (2004) 457-461.

[32] J. Turinsky, B.P. Bayly, D.M. O’Sullivan, 1,2-Diacylglycerol and ceramide levels in rat liver and skeletal muscle in vivo, Am. J. Physiol. 261 (1991) 620-627.

[33] J.K. Kim, J.J. Fillmore, Y. Chen, C. Yu, I.K. Moore, M. Pypaert, et al., Tissuespecific overexpression of lipoprotein lipase causes tissue-specific insulin resistance, Proc. Natl. Acad. Sci. U. S. A. 98 (2001) 7522-7527.

[34] J.M. Adams, T. Pratipanawatr, R. Berria, E. Wang, R.A. DeFronzo, M.C. Sullards, et al., Ceramide content is increased in skeletal muscle from obese insulinresistant humans, Diabetes 53 (2004) 25-31.

[35] C.R. Gault, L.M. Obeid, Y.A. Hannun, An overview of sphingolipid metabolism: from synthesis to breakdown, Adv. Exp. Med. Biol. 688 (2010) 1-23.

[36] J. Rieusset, M.-A. Chauvin, A. Durand, A. Bravard, F. Laugerette, M.C. Michalski, et al., Reduction of endoplasmic reticulum stress using chemical chaperones or Grp78 overexpression does not protect muscle cells from palmitate-induced insulin resistance, Biochem. Biophys. Res. Commun. 417 (2012) 439-445.

[37] S. Turban, E. Hajduch, Protein kinase C isoforms: mediators of reactive lipid metabolites in the development of insulin resistance, FEBS Lett. 585 (2011) 269-274.

[38] A. Kamagate, D.H. Kim, T. Zhang, S. Slusher, R. Gramignoli, S.C. Strom, et al., Oleate blocks palmitate-induced abnormal lipid distribution, endoplasmic reticulum expansion and stress, and insulin resistance in skeletal muscle, Endocrinology 152 (2010) 2206-2218.

[39] S. Fulda, A.M. Gorman, O. Hori, A. Samali, Cellular stress responses: cell survival and cell death, Int. J. Cell Biol. 2010 (2010), 214074.

[40] M. Skelin, M. Rupnik, A. Cencic, Pancreatic beta cell lines and their applications in diabetes mellitus research, ALTEX Alternativen Zu Tierexperimenten 27 (2010) 105-113.

[41] I. Quesada, E. Tudurí, C. Ripoll, A. Nadal, Physiology of the pancreatic alphacell and glucagon secretion: role in glucose homeostasis and diabetes, J. Endocrinol. 199 (2008) 5-19.

[42] T. Ulsen, M.A., Introduction, Chapter 2. Islets, 2000, pp. 33-52.

[43] C. Bernard, M.F. Berthault, C. Saulnier, A. Ktorza, Neogenesis vs. apoptosis as main components of pancreatic beta cell mass changes in glucose-infused normal and mildly diabetic adult rats, FASEB J. 13 (1999) 1195-1205.

[44] T.G. Barrett, S.E. Bundey, Wolfram (DIDMOAD) syndrome, J. Med. Genet. 4 (1997) 838-841.

[45] F. Khanim, J. Kirk, F. Latif, T.G. Barrett, WFS1/wolframin mutations, Wolfram syndrome, and associated diseases, Hum. Mutat. 17 (2001) 357-367.

[46] K. Cryns, T.A. Sivakumaran, J.M. Van den Ouweland, R.J. Pennings, C.W. Cremers, K. Flothmann, T.L. Young, R.J. Smith, M.M. Lesperance, G. Van
Camp, Mutational spectrum of the WFS1 gene in Wolfram syndrome, nonsyndromic hearing impairment, diabetes mellitus, and psychiatric disease, Hum. Mutat. 22 (2003) 275-287.

[47] T. Yamada, H. Ishihara, A. Tamura, R. Takahashi, S. Yamaguchi, D. Takei A. Tokita, C. Satake, F. Tashiro, H. Katagiri, H. Aburatani, J. Miyazaki, Y. Oka, WFS1-deficiency increases endoplasmic reticulum stress, impairs cell cycle progression and triggers the apoptotic pathway specifically in pancreaticcells, Hum. Mol. Genet. 15 (2006) 1600-1609.

[48] M. Zatyka, C. Ricketts, G. da Silva Xavier, J. Minton, S. Fenton, S. HofmannThiel, G.A. Rutter, T. Barrett, Sodium-potassium ATPase 1 subunit is a molecular partner of Wolframin, an endoplasmic reticulum (ER) protein involved in ER-stress, Hum. Mol. Genet. 17 (2008) 190-200.

[49] S. Matsuoka, M. Edwards, C. Bai, S. Parker, P. Zhang, A. Baldini, J. Harper, S. Elledge, p57, a structurally distinct member of the p21 cdk inhibitor family, is a candidate tumor suppressor gene, Genes Dev. 9 (1995) 650662.

[50] Y. Luo, J. Hurwitz, J. Massagué, Cell-cycle inhibition by independent CDK and PCNA binding domains in p21, Nature (Lond.) 375 (1995) 159-161.

[51] J.W. Harper, S.J. Elledge, K. Keyomarsi, B. Dynlacht, L. Tsai, P. Zhang, S. Dobtowolski, C. Bai, L. Connell-Crowley, E. Swindell, M. P Fox, N. Wei, Inhibition of cyclin dependent kinases by p21, Mol. Biol. Cell. 6 (1995) $387-400$.

[52] B.D. Chang, et al., Effects of p21Waf1/Cip1/Sdi1 on cellular gene expression: implications for carcinogenesis, senescence, and age-related diseases, Proc. Natl. Acad. Sci. U. S. A. 97 (2000) 4291-4296.

[53] N.D. Perkins, L.K. Felzien, J.C. Betts, K. Leung, D.H. Beach, G.J. Nabel, Regulation of NF-kappa B by cyclin-dependent kinases associated with the p300 coactivator, Science 275 (1997) 523-527.

[54] J.C. Poole, A. Thain, N.D. Perkins, I.B. Roninson, Induction of transcription by p21Waf1/Cip1/Sdi1: role of NFkappaB and effect of non-steroidal anti-inflammatory drugs, Cell Cycle 3 (2004) 931-940.

[55] A.L. Gartel, S.K. Radhakrishnan, Lost in transcription: p21 repression, mechanisms, and consequences, Cancer Res. 65 (2005) 3980-3985.

[56] D.C. Porter, E. Farmaki, S. Altilia, G.P. Schools, D.K. West, M. Chen, et al., Cyclindependent kinase 8 mediates chemotherapy-induced tumor-promoting paracrine activities, Proc. Natl. Acad. Sci. U. S. A. 109 (2012).

[57] A. L Gartel, A.L. Tyner, A.L. Gartel, A.L. Tyner, The role of the cyclin-dependent kinase inhibitor p21 in apoptosis 1, Mol. Cancer Ther. 1 (2002) 639-649.

[58] T. Cheng, N. Rodrigues, H.M. Shen, Y.G. Yang, D. Dombkowski, M. Sykes, et al. Hematopoietic stem cell quiescence maintained by p21(cip1/waf1), Science 287 (2000) 1804-1808.

[59] H. Hong, K. Takahashi, T. Ichisaka, T. Aoi, O. Kanagawa, M. Nakagawa, et al., Suppression of induced pluripotent stem cell generation by the p53-p21 pathway, Nature 460 (2009) 1132-1135.

[60] H. Kaneto, Y. Kajimoto, Y. Fujitani, T. Matsuoka, K. Sakamoto, M. Matsuhisa, Y. Yamasaki, et al., Oxidative stress induces p21 expression in pancreatic islet cells: possible implication in beta-cell dysfunction, Diabetologia 42 (1999) 1093-1097.

[61] I. Cozar-Castellano, M. Haught, A.F. Stewart, The cell cycle inhibitory protein p21cip is not essential for maintaining beta-cell cycle arrest or beta-cell function in vivo, Diabetes 55 (2006) 3271-3278.

[62] N. Dioufa, I. Chatzistamou, E. Farmaki, A.G. Papavassiliou, H. Kiaris, p53 antagonizes the unfolded protein response and inhibits ground glass hepatocyte development during endoplasmic reticulum stress, Exp. Biol. Med. 237 (2012) 1173-1180.

[63] P. Yesil, E. Lammert, Islet dynamics: a glimpse at beta cell proliferation, Histol Histopathol. 23 (2008) 883-895.

[64] A. Vetere, B.K. Wagner, Chemical methods to induce beta-cell proliferation, Int. J. Endocrinol. (2012) 1-8.

[65] M.L. Graham, J.L. Janecek, J.A. Kittredge, B.J. Hering, H.-J. Schuurman, The streptozotocin-induced diabetic nude mouse model: differences between animals from different sources, Comp. Med. 61 (2011) 356-360.

[66] S. Alpert, D. Hanahan, G. Teitelman, Hybrid insulin genes reveals a developmental lineage for pancreatic endocrine cells and imply a relationship with neurons, Cell 53 (1988) 295-308.

[67] M. Polak, L. Bouchareb-Banaei, R. Scharfmann, P. Czernichow, Early pattern of differentiation in the human pancreas, Diabetes 49 (2000) 225-232.

[68] Y.-P. Fan, R.H. Weiss, Exogenous attenuation of p21(Waf1/Cip1) decreases mesangial cell hypertrophy as a result of hyperglycemia and IGF-1, J. Am. Soc. Nephrol. 15 (2004) 575-584.

[69] T.W. Fawcett, J.L. Martindale, K.Z. Guyton, T. Hai, N.J. Holbrook, Complexes containing activating transcription factor (ATF)/cAMP-responsive-elementbinding protein (CREB) interact with the CCAAT/enhancer-binding protein (C EBP)-ATF composite site to regulate Gadd153 expression during the stress response, Biochem. J. 339 (1999) 135-141.

[70] J. Wu, et al., ATF6alpha optimizes long-term endoplasmic reticulum function to protect cells from chronic stress, Dev. Cell 13 (2007) 351-364.

[71] H. Puthalakath, L.A. O'Reilly, P. Gunn, L. Lee, P.N. Kelly, N.D. Huntington, P.D. Hughes, et al., ER stress triggers apoptosis by activating $\mathrm{BH}$-only protein Bim, Cell 129 (2007) 1337-1349.

[72] S. Oyadomari, et al., Targeted disruption of the CHOP gene delays endoplasmic reticulum stress-mediated diabetes, J. Clin. Invest. 109 (2002) 525-532.

[73] E. Araki, S. Oyadomari, M. Mori, Impact of endoplasmic reticulum stress pathway on pancreatic beta-cells and diabetes mellitus, Exp. Biol. Med. Maywood NJ 228 (2003) 1213-1217. 
[74] Y. Shi, K.M. Vattem, R. Sood, J. An, J. Liang, L. Stramm, R.C. Wek, Identification and characterization of pancreatic eukaryotic initiation factor 2-subunit kinase, PEK, involved in translational control, Mol. Cell. Biol. 18 (1998) 7499-7509.

[75] D. Scheuner, B. Song, E. McEwen, et al., Translational control is required for the unfolded protein response and in vivo glucose homeostasis, Mol. Cell 7 (2001) 1165-1176.

[76] S.H. Back, D. Scheuner, J. Han, et al., Translation attenuation through eIF2 $\alpha$ phosphorylation preserves ER integrity, prevents oxidative stress, and maintains insulin production in $\beta$-cells, Cell Metab. 10 (2009) 1-2.
[77] D.R. Laybutt, A.M. Preston, M.C. Akerfeldt, et al., Endoplasmic reticulum stress contributes to $\beta$-cell apoptosis in type 2 diabetes, Diabetologia 50 (2007) $752-763$.

[78] S.J. Marciniak, D. Ron, Endoplasmic reticulum stress signaling in disease, Physiol. Rev. 86 (2006) 1133-1149.

[79] B. Yusta, L.L. Baggio, J.L. Estall, J.A. Koehler, D.P. Holland, H. Li, et al, GLP-1 receptor activation improves beta cell function and survival following induction of endoplasmic reticulum stress, Cell Metab. 4 (2006) 391-406. 